\title{
Modulation of Inflammation-Induced Tolerance in Cancer
}

\author{
Vladimir Rogovskii * \\ Department of Molecular Pharmacology and Radiobiology, Pirogov Russian National Research Medical University, Moscow, \\ Russia
}

Keywords: cancer, inflammation, immune tolerance, immune suppression, anti-inflammatory therapy

\section{INTRODUCTION}

Chronic inflammation is widely accepted to be an important cause of tumor formation and development $(1,2)$.

The primary significance of chronic inflammation in cancer may be in its linkage to immune tolerance. According to Peter Medawar, "Immunological tolerance' may be described as a state of indifference or non-reactivity toward a substance that would normally be expected to excite an immunological response" (3).

During acute inflammation, immune suppression is a temporary stage toward the resolution of inflammation (4). However, in the case of chronic inflammation, this stage becomes permanent, leading to true immune suppression and immune tolerance. Thus, chronic low-grade inflammation may be the universal mechanism of immune tolerance-both in disease and in health (2). According to this concept, immune tolerance may be the physiological counterpart to chronic lowgrade inflammation [so-called para-inflammation (5)]. This is the case for organs with elevated immune tolerance, e.g., gut, placenta, brain, testis-they have a normally elevated level of several inflammatory factors $(2,5-8)$.

There are various reasons for chronic inflammation: e.g., prolonged contact with irritating factors (physical or chemical), chronic infections, chronic stress, insufficient physical activity, obesity, disturbances in gut microbiota, and an "inflammatory diet" (e.g., a western diet high in processed meat and fat but with low fiber and low omega 3/omega 6 fatty acid ratio) $(2,5,9,10)$. Moreover, in the case of an advanced tumor, such tumors promote an inflammatory environment per se, leading to chronic inflammation (2).

There might be two ways of mitigating chronic inflammation in cancer-intensification of inflammation and suppression of the inflammation. In the case of increasing the inflammation, we intend to surpass inflammation-related tolerance. In the case of suppressing the inflammation, we intend to suppress inflammation-related tolerance, after which there might be tumor rejection (Figure 1).

\section{CANCER-RELATED INFLAMMATION AND IMMUNE TOLERANCE}

Tumors produce several inflammation-related factors, for example, IL-6, IL-8, IL-10, CCL2, and CCL5 $(1,13,14)$.

The current paradigm of the antigen-specific immune suppression is the action of myeloid-derived suppressor cells (MDSCs) (15). According to much studies, this heterogenic cell population may include various dendritic cells, macrophages (M2 macrophages), monocytes and plasmacytoid cells having a similar main feature. They can present antigens to lymphocytes inducing the antigen-specific immune suppression in health (e.g., in immune privileged organs) and disease (16-18). These heterogeneous cell groups express various markers, but the markers from the number of CD11b, CD11c, and CD33 might be the most typical of them $(14,18)$. Lechner et al. revealed that 
induction of CD33+ MDSCs and CD11b + MDSCs depended upon over-expression of various cytokines by a range of different tumor cell lines [especially IL-1 $\beta$, IL-6, TNF $\alpha$, VEGF, GMCSF for CD33+ MDSCs and FMS-like tyrosine kinase 3 ligand (FLT3L), and TGF $\beta$ for CD11b+ MDSCs] (14).

In cancer, MDSCs shape and promote metastatic dissemination (15). Moreover, a low level of MDSCs in patients with cancer has been shown to be correlated with their good prognosis after immune checkpoint inhibitors treatment (19). Among the mechanisms of MDSCs-mediated immune suppression are the production of reactive oxygen species, NO, arginase (Arg-1), indoleamine oxidase (IDO), and immunosuppressive cytokines, such as IL-10, TGF- $\beta$, and T-regulatory cells (Tregs) expansion $(20,21)$.

\section{INCREASING CANCER-RELATED INFLAMMATION}

The first methods for treating cancer by increasing inflammation were made over a century ago by William Coley and many of his followers. In 1891, William Coley injected streptococcal organisms into a patient with inoperable cancer. It was successful, and this was one of the first examples of cancer immunotherapy that enhanced the immune system (22). Another step in the research of increasing cancer-related inflammation was made about 80 years ago by Roskin and colleagues by using Trypanosoma cruzi (the protozoan agent of Chagas' disease). It was proposed that this infection mediates an adaptive immune response with significant antitumor effects (23).

Many attempts were made later to promote the antitumor immune response by increasing inflammation using other approaches.

In particular, cytokine therapy [e.g., IL-2, interferon (IFN)- $\alpha$ ], antitumor vaccines, and even oncolytic viruses (e.g., genetically modified type I herpes simplex virus) were used. Currently, the most widespread way of increasing cancer-related inflammation is the use of immune checkpoint inhibitors (ICIs) and chimeric antigen receptor $\mathrm{T}$ (CAR-T) cells $(11,12,24-28)$.

One of the most commonly used cytokines in cancer treatment is IL-2. IL-2 promotes antigen-activated CD8+ Tcells and is a growth factor for CD4+ T-cells as well as NK-cells. IL-2 has some proven efficacy in treating metastatic renal cell carcinoma and metastatic melanoma (MM) patients (26). The meta-analysis showed that IL-2 immunotherapy for advanced MM delivered a complete response rate of 4\% (29). Besides the immune stimulation, IL-2 plays an essential role in immune suppression as part of a negative feedback loop cascade. IL-2 is a potent negative immune regulator stimulating immunosuppressive Tregs (26). That immune-suppressive role of cytokine signaling might be the cause of poor clinical results.

Oncolytic viruses are another type of inflammation-enhancing immune therapy. They are designed to target and kill cancer cells, leaving normal cells unaffected (30). For example, the modified oncolytic Herpes simplex virus 1 (HSV-1), talimogene laherparepvec (T-VEC), has been shown to suppress the growth
1. Highly activated state

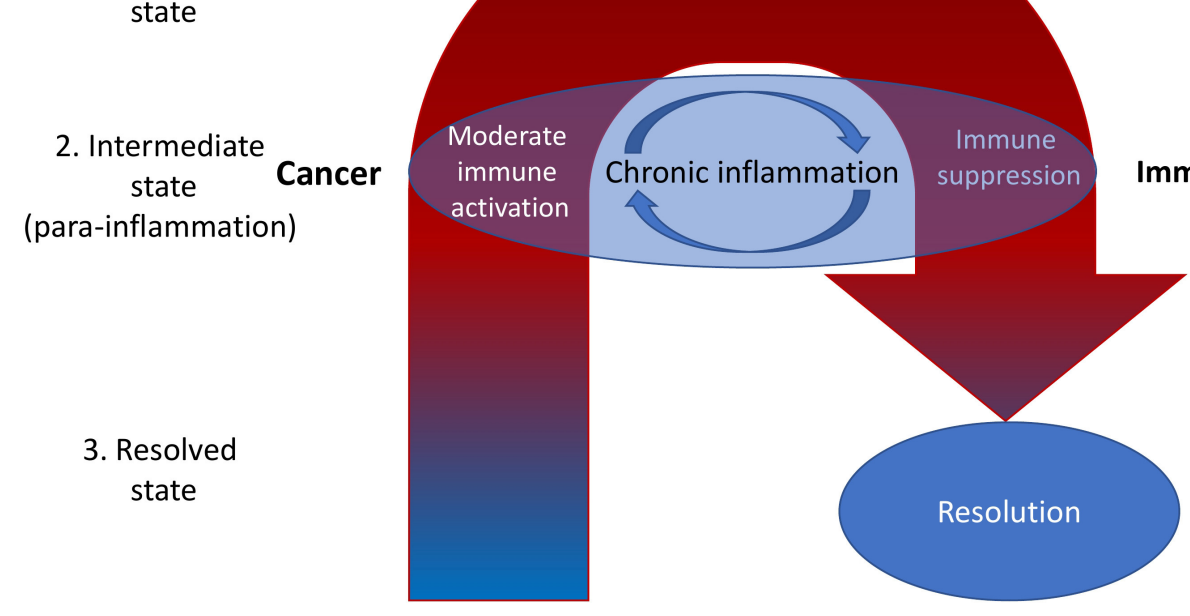

FIGURE 1 | It is reasonable to highlight the three types of inflammatory microenvironments: highly activated state, intermediate state, and the resolved state. Cancer is often associated with the intermediate state [para-inflammation (6)]. Most current therapeutic efforts are directed toward increasing inflammation toward the highly activated state. However, this is often associated with internal compensative mechanisms resulting in subsequent immune suppression (negative feedback). That may be the reason for the limited efficiency of ICls, which dramatically increase inflammation at the whole-organism level (11). A better way may be to reduce inflammation with the aim of subsequent activation of the normal process of immune rejection of tumors (fast and without entering the cycle of chronic inflammation)-it might be called the immunological reload. Nevertheless, there still may be non-immune factors in the tumor microenvironment that decrease the immune response. For example, high levels of oxidized low-density lipoproteins and lactate (12). They also should be eliminated. 
of advanced malignant melanoma in humans (31). It is the first approved oncolytic virus in the USA (2015). In the phase III trial of T-VEC, the objective response rate and complete response rate were 26 and $11 \%$, respectively, compared to 6 and $1 \%$ for recombinant GM-CSF (32). Besides the direct killing of cancer cells, oncolytic viruses can modulate the tumor microenvironment toward a more inflammatory phenotype and induce anti-cancer immunity (30). These processes are very complicated, as there are multiple negative feedback mechanisms. For example, it was shown that chronic viral infection could enhance NK-cells function. This effect is mediated by type I IFN signaling, and it can lead to the killing of virus-specific T cells. The biological sense of this is to minimize T-cell-mediated pathologic damage (33). Minimizing the T-cellmediated response can limit cancer cell killing by $\mathrm{T}$ cells.

It should be taken into account that any induction of inflammatory phenotype leads to a compensatory antiinflammatory and immune-suppressive response sooner or later. In that stage, after the initial reduction of tumor volume, cancer cells might start to proliferate more extensively.

ICIs block signaling through inhibiting receptors in immune cells. The first checkpoint inhibitor was approved in 2011, opening a new era in cancer immunotherapy. Typically, ICIs increase inflammation at the whole organism level (34). This increment at the initial stage can be associated with increased inflammation-related immune tolerance and might be the reason for tumor pseudoprogression. After the predomination of the immune-inflammatory process over immune tolerance, there may be clinical remission.

It should be noted that there are multiple mechanisms of negative feedback in immunity, such as MDSCs, Tregs, and many immune checkpoints (besides CTLA-4 and PD-1, there are TIM-3: mucin-domain-containing protein-3, LAG-3: lymphocyte-activated gene-3, and many others). Moreover, this potent immune-suppressive machinery tends to be activated by increased ICIs-mediated or CAR-T-mediated immune inflammation. That might be the reason why, after an initial response to checkpoint blockade, acquired resistance occurs in most patients (35). The phenomenon of hyperprogression (paradoxical acceleration in tumor growth observed in certain patients following the administration of immune checkpoint inhibitors) also can be linked to these mechanisms (34). In line with them, it was recently found that the percentage of CD8-T-cells that express LAG-3 and PD-1 was significantly increased in the dysfunctional response group to CAR T-cell therapy (36).

\section{REDUCING CANCER-RELATED INFLAMMATION}

Mechanisms of resolution of inflammation are of vital importance for cancer prevention. Animals lacking in immunosuppressive mediators show chronic inflammation and increased cancer frequency $(37,38)$. Anti-inflammatory strategies for cancer treatment include the use of alltrans-retinoic acid (ATRA), vitamin D, non-steroidal anti-inflammatory drugs (NSAIDs), several anti-inflammatory antibodies, etc.

ATRA is the primary biologically active metabolite of vitamin A that possesses anti-inflammatory properties (39). ATRA is crucial for dendritic cells to facilitate the generation of Tregs and suppress the differentiation of naive CD4+ cells into inflammatory Th17-cells (40). ATRA also influences the maturation of MDSCs by increasing the expression of major histocompatibility complex class II and CD86 (41).

It is reasonable to suppose that termination of inflammation (resolution) should also cause the termination of the action of immune-suppressive mechanisms.

For instance, it was shown that in vitro treatment with ATRA decreases the immunosuppressive function of MDSCs in mixed lymphocyte reactions. ATRA also reduces the expression of immunosuppressive genes, including PD-L1, IL-10, and IDO, by MDSCs. In a randomized phase II clinical trial, ATRA significantly decreased the frequency of circulating MDSCs compared to ipilimumab treatment alone in advanced-stage melanoma patients (42). Currently, there are several ongoing trials exploring ATRA in cancer treatment $(43,44)$.

Vitamin D (e.g., calcitriol) exhibits the anti-inflammatory actions that may contribute to its beneficial effects in several cancers. Calcitriol is involved in the inhibition of the synthesis of prostaglandins, suppression of stress-activated kinase signaling, suppression of NF- $\mathrm{B}$ signaling, and other anti-inflammatory mechanisms (45). MDSCs are considered to be one of the important vitamin $\mathrm{D}$ targets. It was shown that vitamin $\mathrm{D}$ treatment reduced the T-cell suppressive capacity of cytokineinduced MDSCs by $\geq 70 \%$ (46).

There are various data concerning vitamin $\mathrm{D}$ and cancer incidence and treatment. For instance, it was shown that vitamin $\mathrm{D}$ was associated with a significant reduction of cancer-related mortality compared with placebo (response rate 0.87 ) (47).

Polyphenols and related compounds are plant metabolites that contain multiple phenolic groups. Polyphenols possess various anti-inflammatory properties. Due to their molecular structure, they can serve as hydrogen bond donors to many proteins and nucleic acids. That is why there are various molecular targets of polyphenols, mainly in the signal transduction pathways $(48,49)$.

For instance, in prostate cancer cell lines, polyphenol resveratrol reduced the levels of several receptor tyrosine kinases (e.g., epidermal growth factor receptor) (50). Catechins (the water-soluble polyphenolic substances found in green tea) also can interact with the ATP binding site of some receptor tyrosine kinases and inhibit tyrosine phosphorylation $(48,51)$. Resveratrol induces autophagy by directly inhibiting mTOR through ATP competition (52).

According to a recent 5-year trial, oral administration of indole-3-carbinol [plant metabolite, obtained from cruciferous vegetables (53)] and epigallocatechin-3-gallate (probably the most active of catechins) as maintenance therapeutic agents in advanced ovarian cancer has demonstrated a dramatic increase in median progression-free survival (approximately double) (54).

Of course, we should discuss the most widely used antiinflammatory drugs-NSAIDs (e.g., aspirin). Their ability to prevent cancer has been known for years. According to a 
2018 meta-analysis, a protective effect was identified for the intake of any NSAIDs against the risk of prostate cancer (55). According to another recent study, regular use of cyclooxygenase- 2 inhibitors was associated with a $71 \%$ reduced risk of breast cancer (56). NSAIDs are involved in modulation of the synthesis of various eicosanoids. It is not just inhibition, but a more complicated process during which synthesis increases of cyclooxygenase- and lipoxygenase-derived anti-inflammatory factors like lipoxin A4, resolvin D1, etc. Eicosanoid mediators are involved in the induction and maintenance of immune tolerance. For instance, lipoxin A4 is involved in tolerance induction during allergen immunotherapy (57). According to a recent study, colorectal cancer is associated with a deficiency of lipoxin A4 (58). In murine xenograft tumor models, lipoxin A4 is able to suppress tumor growth by targeting immune-suppressive IL-10-producing regulatory B cells via dephosphorylation of STAT-3 and extracellular signal-regulated kinase (59).

\section{CONCLUSION AND PROSPECTS}

The efficacy of inflammation-increasing immunotherapy depends on the cancer type (more suitable for "hot" tumors, i.e., those that show signs of inflammation) and is limited by major

\section{REFERENCES}

1. Landskron G, De la Fuente M, Thuwajit P, Thuwajit C, Hermoso MA. Chronic inflammation and cytokines in the tumor microenvironment. J Immunol Res. (2014) 2014:149185. doi: 10.1155/2014/149185

2. Rogovskii VS. The linkage between inflammation and immune tolerance: interfering with inflammation in cancer. Curr Cancer Drug Targets. (2017) 17:325-32. doi: 10.2174/1568009617666170109110816

3. Peter Medawar - Nobel Lecture. NobelPrize.org: Nobel Media AB 2019. Available online at: https://www.nobelprize.org/prizes/medicine/1960/ medawar/lecture/ (accessed November 20, 2019).

4. Serhan CN, Levy BD. Resolvins in inflammation: emergence of the proresolving superfamily of mediators. J Clin Invest. (2018) 128:2657-69. doi: 10.1172/JCI97943

5. Medzhitov R. Origin and physiological roles of inflammation. Nature. (2008) 454:428-35. doi: 10.1038/nature07201

6. Brann E, Edvinsson A, Rostedt Punga A, Sundstrom-Poromaa I, Skalkidou A. Inflammatory and anti-inflammatory markers in plasma: from late pregnancy to early postpartum. Sci Rep. (2019) 9:1863. doi: 10.1038/s41598-018-38304-w

7. Loveland KL, Klein B, Pueschl D, Indumathy S, Bergmann M, Loveland $\mathrm{BE}$, et al. Cytokines in male fertility and reproductive pathologies: immunoregulation and beyond. Front Endocrinol. (2017) 8:307. doi: 10.3389/fendo.2017.00307

8. Mor G, Cardenas I, Abrahams V, Guller S. Inflammation and pregnancy: the role of the immune system at the implantation site. Ann NY Acad Sci. (2011) 1221:80-7. doi: 10.1111/j.1749-6632.2010.05938.x

9. DiNicolantonio JJ, O’Keefe JH. Importance of maintaining a low omega6/omega-3 ratio for reducing inflammation. Open Heart. (2018) 5:e000946. doi: 10.1136/openhrt-2018-000946

10. Kozhieva MK, Melnikov MV, Rogovsky VS, Oleskin AV, Kabilov MR, Boyko AN. Gut human microbiota and multiple sclerosis. Zh Nevrol Psikhiatr Im S S Korsakova. (2017) 117:11-9. doi: 10.17116/jnevro201711710211-19

11. Dwyer CJ, Knochelmann HM, Smith AS, Wyatt MM, Rangel Rivera GO, Arhontoulis DC, et al. Fueling cancer immunotherapy with common gamma chain cytokines. Front Immunol. (2019) 10:263. doi: $10.3389 /$ fimmu.2019.00263 side effects. Active inflammation is often associated with internal compensative mechanisms resulting in possible subsequent immune suppression-it may be one of the reasons for the low response to tumor immunotherapy.

Much data exists regarding the efficiency of suppressing cancer-related inflammation. The aim of this suppression should be to abrogate immune tolerance associated with chronic inflammation. It is probably reasonable to study combination therapy: anti-inflammatory therapy with immunotherapy such as ICIs and CAR-T cells. Such a combination seems contradictory. However, just as one often needs to combine different directions to reach a certain point, e.g., turn left and turn right depending on the current route, cancer immune therapy could require a similar twist.

\section{AUTHOR CONTRIBUTIONS}

VR conceived and wrote the article, read, and approved the submitted version.

\section{ACKNOWLEDGMENTS}

The author thanks Brian Chan for his careful reading that improved the manuscript style.

12. Marchini A, Daeffler L, Pozdeev VI, Angelova A, Rommelaere J. Immune conversion of tumor microenvironment by oncolytic viruses: the protoparvovirus H-1PV case study. Front Immunol. (2019) 10:1848. doi: 10.3389/fimmu.2019.01848

13. Levina V, Su Y, Nolen B, Liu X, Gordin Y, Lee M, et al. Chemotherapeutic drugs and human tumor cells cytokine network. Int J Cancer. (2008) 123:2031-40. doi: $10.1002 /$ ijc. 23732

14. Lechner MG, Megiel C, Russell SM, Bingham B, Arger N, Woo T, et al. Functional characterization of human $\mathrm{Cd} 33+$ and $\mathrm{Cd} 11 \mathrm{~b}+$ myeloid-derived suppressor cell subsets induced from peripheral blood mononuclear cells co-cultured with a diverse set of human tumor cell lines. J Transl Med. (2011) 9:90. doi: 10.1186/1479-58 76-9-90

15. Trovato R, Cane S, Petrova V, Sartoris S, Ugel S, De Sanctis F. The engagement between MDSCs and metastases: partners in crime. Front Oncol. (2020) 10:165. doi: 10.3389/fonc.2020.00165

16. Ponomarev AV, Shubina IZ. Insights into mechanisms of tumor and immune system interaction: association with wound healing. Front Oncol. (2019) 9:1115. doi: 10.3389/fonc.2019.01115

17. Elliott LA, Doherty GA, Sheahan K, Ryan EJ. Human tumor-infiltrating myeloid cells: phenotypic and functional diversity. Front Immunol. (2017) 8:86. doi: 10.3389/fimmu.2017.00086

18. Goubier A, Dubois B, Gheit H, Joubert G, Villard-Truc F, Asselin-Paturel C, et al. Plasmacytoid dendritic cells mediate oral tolerance. Immunity. (2008) 29:464-75. doi: 10.1016/j.immuni.2008.06.017

19. Park SM, Youn JI. Role of myeloid-derived suppressor cells in immune checkpoint inhibitor therapy in cancer. Arch Pharm Res. (2019) 42:560-6. doi: 10.1007/s12272-019-01165-6

20. Wang Y, Ding Y, Guo N, Wang S. MDSCs: key criminals of tumor pre-metastatic niche formation. Front Immunol. (2019) 10:172. doi: 10.3389/fimmu.2019.00172

21. Salminen A, Kaarniranta K, Kauppinen A. The role of myeloid-derived suppressor cells (MDSC) in the inflammaging process. Ageing Res Rev. (2018) 48:1-10. doi: 10.1016/j.arr.2018.09.001

22. McCarthy EF. The toxins of William B. Coley and the treatment of bone and soft-tissue sarcomas. Iowa Orthop J. (2006) 26:154-8. 
23. Ramirez-Toloza G, Abello P, Ferreira A. Is the antitumor property of Trypanosoma cruzi infection mediated by its calreticulin? Front Immunol. (2016) 7:268. doi: 10.3389/fimmu.2016.00268

24. Perez CR, De Palma M. Engineering dendritic cell vaccines to improve cancer immunotherapy. Nat Commun. (2019) 10:5408. doi: 10.1038/s41467-019-13368-y

25. Conry RM, Westbrook B, McKee S, Norwood TG. Talimogene laherparepvec: first in class oncolytic virotherapy. Hum Vaccin Immunother. (2018) 14:83946. doi: 10.1080/21645515.2017.1412896

26. Conlon KC, Miljkovic MD, Waldmann TA. Cytokines in the treatment of cancer. J Interferon Cytokine Res. (2019) 39:6-21. doi: 10.1089/jir.2018.0019

27. Marhelava K, Pilch Z, Bajor M, Graczyk-Jarzynka A, Zagozdzon R. Targeting negative and positive immune checkpoints with monoclonal antibodies in therapy of cancer. Cancers. (2019) 11:1756. doi: 10.3390/cancers 11111756

28. Martinez M, Moon EK. CAR T cells for solid tumors: new strategies for finding, infiltrating, and surviving in the tumor microenvironment. Front Immunol. (2019) 10:128. doi: 10.3389/fimmu.2019.00128

29. Bright R, Coventry BJ, Eardley-Harris N, Briggs N. Clinical response rates from interleukin-2 therapy for metastatic melanoma over 30 years' experience: a meta-analysis of 3312 patients. J Immunother. (2017) 40:21-30. doi: 10.1097/CJI.0000000000000149

30. Ylosmaki E, Cerullo V. Design and application of oncolytic viruses for cancer immunotherapy. Curr Opin Biotechnol. (2019) 65:25-36. doi: 10.1016/j.copbio.2019.11.016

31. Valyi-Nagy T, Fredericks B, Ravindra A, Hopkins J, Shukla D, Valyi-Nagy K. Herpes simplex virus 1 infection promotes the growth of a subpopulation of tumor cells in three-dimensional uveal melanoma cultures. J Virol. (2018) 92:e00700-18. doi: 10.1128/JVI.00700-18

32. Eissa IR, Bustos-Villalobos I, Ichinose T, Matsumura S, Naoe Y, Miyajima N, et al. The current status and future prospects of oncolytic viruses in clinical trials against melanoma, glioma, pancreatic, and breast cancers. Cancers. (2018) 10:356. doi: 10.3390/cancers10100356

33. Oh JH, Kim MJ, Choi SJ, Ban YH, Lee HK, Shin EC, et al. Sustained type I interferon reinforces NK cell-mediated cancer immunosurveillance during chronic virus infection. Cancer Immunol Res. (2019) 7:584-99. doi: 10.1158/2326-6066.CIR-18-0403

34. Denis M, Duruisseaux M, Brevet M, Dumontet C. How can immune checkpoint inhibitors cause hyperprogression in solid tumors? Front Immunol. (2020) 11:492. doi: 10.3389/fimmu.2020.00492

35. Barrueto L, Caminero F, Cash L, Makris C, Lamichhane P, Deshmukh RR. Resistance to checkpoint inhibition in cancer immunotherapy. Transl Oncol. (2020) 13:100738. doi: 10.1016/j.tranon.2019.12.010

36. Xu X, Sun Q, Liang X, Chen Z, Zhang X, Zhou X, et al. Mechanisms of relapse after CD19 CAR T-cell therapy for acute lymphoblastic leukemia and its prevention and treatment strategies. Front Immunol. (2019) 10:2664. doi: 10.3389/fimmu.2019.02664

37. Qu X, Tang Y, Hua S. Immunological approaches towards cancer and inflammation: a cross talk. Front Immunol. (2018) 9:563. doi: 10.3389/fimmu.2018.00563

38. Wu F, Weigel KJ, Zhou H, Wang XJ. Paradoxical roles of TGF-beta signaling in suppressing and promoting squamous cell carcinoma. Acta Biochim Biophys Sin. (2018) 50:98-105. doi: 10.1093/abbs/gmx127

39. Uniyal S, Dhasmana A, Tyagi A, Muyal JP. ATRA reduces inflammation and improves alveolar epithelium regeneration in emphysematous rat lung. Biomed Pharmacother. (2018) 108:1435-50. doi: 10.1016/j.biopha.2018.09.166

40. Liu ZM, Wang KP, Ma J, Guo Zheng S. The role of all-trans retinoic acid in the biology of Foxp3+ regulatory T cells. Cell Mol Immunol. (2015) 12:553-7. doi: $10.1038 / \mathrm{cmi} .2014 .133$

41. Rios FJ, Moustaid-Moussa N, Martins JO. Interplay between hormones, the immune system, and metabolic disorders. Mediators Inflamm. (2018) 2018:8654212. doi: 10.1155/2018/8654212

42. Tobin RP, Jordan KR, Robinson WA, Davis D, Borges VF, Gonzalez R, et al. Targeting myeloid-derived suppressor cells using all-trans retinoic acid in melanoma patients treated with Ipilimumab. Int Immunopharmacol. (2018) 63:282-91. doi: 10.1016/j.intimp.2018.08.007

43. ClinicalTrials.gov. A Trial of All-trans Retinoic Acid (ATRA) in Advanced Adenoid Cystic Carcinoma. Available online at: https://clinicaltrials.gov/ct2/ show/NCT03999684 (accessed March 8, 2020).
44. ClinicalTrials.gov. Available online at: https://clinicaltrials.gov/ct2/show/ NCT04113863 (accessed March 8, 2020).

45. Krishnan AV, Trump DL, Johnson CS, Feldman D. The role of vitamin $\mathrm{D}$ in cancer prevention and treatment. Endocrinol Metab Clin North Am. (2010) 39:401-18. doi: 10.1016/j.ecl.2010.02.011

46. Fleet JC, Burcham GN, Calvert RD, Elzey BD, Ratliff TL. 1alpha, 25 Dihydroxyvitamin D (1,25(OH)2D) inhibits the T cell suppressive function of myeloid derived suppressor cells (MDSC). J Steroid Biochem Mol Biol. (2019) 198:105557. doi: 10.1016/j.jsbmb.2019.105557

47. Haykal T, Samji V, Zayed Y, Gakhal I, Dhillon H, Kheiri B, et al. The role of vitamin $\mathrm{D}$ supplementation for primary prevention of cancer: meta-analysis of randomized controlled trials. J Community Hosp Intern Med Perspect. (2019) 9:480-8. doi: 10.1080/20009666.2019.1701839

48. Rogovskii VS, Popov SV, Sturov NV, Shimanovskii NL. The possibility of preventive and therapeutic use of green tea catechins in prostate cancer. Anticancer Agents Med Chem. (2019) 19:1223-31. doi: 10.2174/1871520619666190404153058

49. Fedotcheva TA, Matyushin AI, Rzheznikov VM, Shimanovskii NL. Antioxidant and cytoprotector properties of genistein - isoflavone with estrogenic activity. Eksp Klin Farmakol. (2016) 79:24-8. doi: 10.30906/0869-2092-2016-79-12-24-28

50. Costea T, Nagy P, Ganea C, Szollosi J, Mocanu MM. Molecular mechanisms and bioavailability of polyphenols in prostate cancer. Int J Mol Sci. (2019) 20:1062. doi: 10.3390/ijms20051062

51. Yang CS, Wang H, Chen JX, Zhang J. Effects of tea catechins on cancer signaling pathways. Enzymes. (2014) 36:195-221. doi: 10.1016/B978-0-12-802215-3.00010-0

52. Park D, Jeong H, Lee MN, Koh A, Kwon O, Yang YR, et al. Resveratrol induces autophagy by directly inhibiting mTOR through ATP competition. Sci Rep. (2016) 6:21772. doi: 10.1038/srep21772

53. Maruthanila VL, Poornima J, Mirunalini S. Attenuation of carcinogenesis and the mechanism underlying by the influence of indole-3-carbinol and its metabolite 3,3'-diindolylmethane: a therapeutic marvel. Adv Pharmacol Sci. (2014) 2014:832161. doi: 10.1155/2014/832161

54. Kiselev VI, Ashrafyan LA, Muyzhnek EL, Gerfanova EV, Antonova IB, Aleshikova OI, et al. A new promising way of maintenance therapy in advanced ovarian cancer: a comparative clinical study. BMC Cancer. (2018) 18:904. doi: 10.1186/s12885-018-4792-9

55. Shang Z, Wang X, Yan H, Cui B, Wang Q, Wu J, et al. Intake of nonsteroidal anti-inflammatory drugs and the risk of prostate cancer: a meta-analysis. Front Oncol. (2018) 8:437. doi: 10.3389/fonc.2018. 00437

56. Kehm RD, Hopper JL, John EM, Phillips KA, MacInnis RJ, Dite GS, et al. Regular use of aspirin and other non-steroidal anti-inflammatory drugs and breast cancer risk for women at familial or genetic risk: a cohort study. Breast Cancer Res. (2019) 21:52. doi: 10.1186/s13058-0191135-y

57. Lech Z, Chalubinski M, Kosinski S, Smorawska E, Bassin C, Akdis CA, et al. Prostaglandin E2 and lipoxin A4 in PBMCs are associated with immune tolerance during venom immunotherapy. J Allergy Clin Immunol. (2016) 138:1199-202 e2. doi: 10.1016/j.jaci.2016.03.024

58. Liu H, Zeng J, Huang W, Xu Q, Ye D, Sun R, et al. Colorectal cancer is associated with a deficiency of lipoxin A4, an endogenous anti-inflammatory mediator. J Cancer. (2019) 10:4719-30. doi: 10.7150/jca.32456

59. Zhang Q, Zhu B, Li Y. Resolution of cancer-promoting inflammation: a new approach for anticancer therapy. Front Immunol. (2017) 8:71. doi: $10.3389 /$ fimmu.2017.00071

Conflict of Interest: The author declares that the research was conducted in the absence of any commercial or financial relationships that could be construed as a potential conflict of interest.

Copyright (c) 2020 Rogovskii. This is an open-access article distributed under the terms of the Creative Commons Attribution License (CC BY). The use, distribution or reproduction in other forums is permitted, provided the original author(s) and the copyright owner(s) are credited and that the original publication in this journal is cited, in accordance with accepted academic practice. No use, distribution or reproduction is permitted which does not comply with these terms. 